\title{
5 A FELICIDADE COMO CATALISADORA DOS NÍVEIS DE ANSIEDADE, DEPRESSÃO E STRESS NO ENSINO SUPERIOR
}

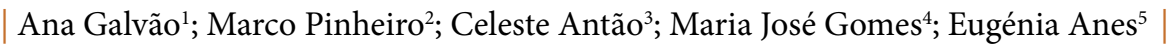

\section{RESUMO}

CONTEXTO: A felicidade como medida do bem-estar, tem vindo a ganhar importância na última década. Contudo, o mundo moderno, impõe cada vez mais exigências aos indivíduos, promovendo altos níveis de ansiedade, depressão e stress, que por sua vez podem influenciar negativamente a felicidade.

OBJETIVO(S): Identificar os níveis de felicidade, ansiedade, depressão e stress dos docentes, pessoal não docente e estudantes do ensino superior; identificar relações entre a felicidade e a ansiedade, depressão e stress.

MÉTODOS: Estudo transversal, descritivo, correlacional e inferencial. Amostra de 732 indivíduos de institutos politécnicos: 189 docentes; 121 pessoal não docente; e 422 estudantes. Utilizou-se um questionário online, com questões socioprofissionais, uma parte com o Oxford Happiness Questionnaire, e a terceira parte com a Escala de Ansiedade, Depressão e Stress.

RESULTADOS: Verificou-se que o cluster dos alunos é o que apresenta menores níveis de felicidade e níveis superiores de Ansiedade, Depressão e Stress. O grupo com níveis mais elevados de felicidade é dos docentes $(\mathrm{M}=4.26 ; \mathrm{DP}=.663)$, bem como em termos de Ansiedade $(\mathrm{M}=2.56$; $\mathrm{DP}=3.022)$, Depressão $(\mathrm{M}=3.32 ; \mathrm{DP}=3.667)$ e Stress $(\mathrm{M}=5.94$; $\mathrm{DP}=4.109)$. Verificaram-se diferenças estatisticamente significativas para a felicidade entre os grupos e correlações negativas baixas a moderadas entre o nível de felicidade e as dimensões de saúde mental sob estudo.

CONCLUSÕES: Verificou-se uma relação entre os níveis de ansiedade, depressão e stress e o nível de felicidade. Propomos que este estudo deverá ser aprofundado para identificar as possíveis causas que provocam o baixo nível de felicidade e os altos níveis de ansiedade, depressão e stress dos estudantes.

PALAVRAS-CHAVE: Felicidade; Ansiedade; Depressão; Stress psicológico

\section{RESUMEN}

"La felicidad como catalizador de los niveles de ansiedad, depresión y estrés en la enseñanza superior"

CONTEXTO: La felicidad como medida del bienestar, ha venido a ganar importancia en la última década. Sin embargo, el mundo moderno, impone cada vez más exigencias a los individuos, promoviendo altos niveles de ansiedad, depresión y estrés, que a su vez pueden influenciar negativamente la felicidad. OBJETIVO(S): Identificar los niveles de felicidad, ansiedad, depresión y estrés de los docentes, personal no docente y estudiantes de la enseñanza politécnica; identificar relaciones entre la felicidad y la ansiedad, la depresión y el estrés. METODOLOGÍA: Estudio transversal, descriptivo, correlacional e inferencial. Muestra de 732 individuos de enseñanza superior: 189 docentes; 121 personal no docente; y 422 estudiantes. Se utilizó un cuestionario online, con cuestiones socioprofesionales, una parte con el Oxford Happiness Questionnaire, y la tercera parte con la Escala de Ansiedad, Depresión y Stress.

RESULTADOS: Se verificó que los alumnos son los que presentan menores niveles de felicidad y niveles superiores de Ansiedad, Depresión y Stress. El grupo con mejores niveles de felicidad es el grupo de los docentes $(M=4.26$, $\mathrm{DP}=.663)$, así como en términos de Ansiedad $(\mathrm{M}=2.56$, $\mathrm{DP}=3.022)$, Depresión $(\mathrm{M}=3.32, \mathrm{DP}=3.667)$ Stress $(\mathrm{M}=5.94, \mathrm{DP}=4.109)$. Se verificaron diferencias estadísticamente significativas para la felicidad entre varios grupos y correlaciones negativas bajas a moderadas entre el nivel de felicidad y las dimensiones de salud mental bajo estudio.

CONCLUSIONES: Se verificó una relación entre los niveles de ansiedad, depresión y estrés y el nivel de felicidad. Proponemos que este estudio debe profundizarse para identificar las posibles causas que provocan el bajo nivel de felicidad y los altos niveles de ansiedad, depresión y estrés de los estudiantes.

\section{DESCRIPTORES: Felicidad; Ansiedad; Depresión; Estrés psi- cológico}

\section{ABSTRACT \\ "Happiness as a catalyst for anxiety, depression and stress levels in higher education"}

BACKGROUND: Happiness as a measure of well-being has been gaining importance in the last decade. However, the modern world imposes more and more demands on individuals, promoting high levels of anxiety, depression and stress, which in turn can negatively influence happiness.

AIM: To identify the levels of happiness, anxiety, depression and stress of teachers, non-teaching staff and students of higher education; to identify relationships between happiness and anxiety, depression and stress.

METHODS: Cross-sectional, descriptive, correlational and inferential study. Sample of 732 individuals from polytechnic institutes: 189 teachers; 121 nonteaching staff; and 422 students. An online questionnaire was used, with socioprofessional questions, one part with the Oxford Happiness Questionnaire, and the third with the Anxiety, Depression and Stress Scale.

RESULTS: It was verified that the students are those with lower levels of happiness and higher levels of Anxiety, Depression and Stress. The group with the highest levels of happiness is the group of teachers $(\mathrm{M}=4.26, \mathrm{SD}=.663)$, as well as in terms of Anxiety $(M=2.56, S D=3.022)$, Depression $(M=3.32, S D$ $=$ Stress $(M=5.94, S D=4.109)$. There were statistically significant differences for happiness among several of the groups and low to moderate negative correlations between the level of happiness and the dimensions of mental health under study.

CONCLUSIONS: There was a relationship between levels of anxiety, depression and stress and the level of happiness. We propose that this study should be deepened to identify the possible causes that cause the low level of happiness and the high levels of anxiety, depression and stress of the students.

KEYWORDS: Happiness; Anxiety; Depression; Psychological stress

Submetido em 30-12-2018

Aceite em 20-02-2019

1 Doutora; Psicóloga, Investigadora no Núcleo UICISA:E da Escola Superior de Saúde; Professora Coordenadora no Instituto Politécnico de Bragança, anagalvao@ipb.pt 2 Licenciado; Especialista em Gestão Empresarial; Docente Especialmente Convidado no Instituto Politécnico de Bragança, Portugal, mpinheiro@ipb.pt

3 Doutora; Enfermeira especialista em Enfermagem de Saúde Infantil e Pediátrica; Investigadora no Núcleo UICISA:E da Escola Superior de Saúde; Professora Adjunta no Instituto Politécnico de Bragança, Portugal, celeste@ipb.pt

4 Doutora; Enfermeira especialista em Enfermagem de Reabilitação; Investigadora no Núcleo UICISA:E da Escola Superior de Saúde; Professora Adjunta no Instituto Politécnico de Bragança, Portugal, mgomes@ipb.pt

5 Doutora; Enfermeira especialista em Enfermagem Comunitária; Investigadora no Núcleo UICISA:E da Escola Superior de Saúde; Professora Adjunta no Instituto Politécnico de Bragança, Portugal, eugenia@ipb.pt

Citação: Galvão, A., Pinheiro, M., Antão, C., Gomes, M. J., \& Anes, E. (2020). A felicidade como catalisadora dos níveis de ansiedade, depressão e stress no ensino superior. Revista Portuguesa de Enfermagem de Saúde Mental (Spe7), 31-35. 


\section{INTRODUÇÃO}

A felicidade como medida do bem-estar, tem vindo a ganhar importância nas últimas décadas, angariando não só o interesse na psicologia, mas também noutras áreas, como por exemplo a economia, onde inclusivamente há cada vez mais países a calcularem a Felicidade Interna Bruta além dos tradicionais dados económicos do país. Contudo, o mundo moderno impõe cada vez mais exigências aos indivíduos, promovendo altos níveis de ansiedade, depressão e stress, que por sua vez podem influenciar negativamente a felicidade e consequentemente o bem-estar.

A felicidade é interpretada de várias formas na literatura relacionando-se com a satisfação com a vida, a alegria, o prazer, e/ ou o bem-estar subjetivo (Jalloh, Flack, \& Chen, 2014). Outros estudos indicam que a felicidade pode ser classificada em três dimensões, sendo estas: a frequência e grau de comprometimento positivo; o nível médio de satisfação com a vida durante um período de tempo; e, a ausência de emoções negativas, como a ansiedade ou a depressão ( $\mathrm{Lu} \&$ Argyle, 1994). Existem evidências que a felicidade é uma das principais componentes da qualidade de vida em todas as idades, promovendo uma atitude positiva perante a vida, um autoconceito positivo, esperança, tentativa de alcançar metas, estabilidade nas relações sociais, satisfação com os outros, melhoria do trabalho em equipa e, em geral, uma melhoria da saúde mental (Lyubomirsky, Tkach, \& DiMatteo, 2006). Além da saúde física e o sistema imunológico serem positivamente afetados pela felicidade foi encontrada uma correlação positiva entre a felicidade e o desempenho académico e/ou profissional. A felicidade reduz o esgotamento, principalmente em trabalhos stressantes (Keramati, 2014).

\section{MÉTODOS}

Objetivou-se identificar os níveis de felicidade, ansiedade, depressão e stress dos docentes, pessoal não docente e estudantes do ensino superior e possíveis diferenças entre grupos. Simultaneamente, identificar relações entre a felicidade e a ansiedade, depressão e stress.

Procedeu-se a um estudo transversal, descritivo, correlacional e inferencial, numa amostra de 732 indivíduos de institutos politécnicos (no caso das Universidades dos Açores e Aveiro, os questionários só foram preenchidos por indivíduos do ensino superior politécnico que integram essas universidades), dos quais
703 (96\%) portugueses, sendo 189 (25.8\%) docentes, 121 (16.5\%) pessoal não docente e 422 (57.7\%) estudantes, conforme apresentado na Tabela 1 .

Tabela 1 - Caraterização Socioprofissional

\begin{tabular}{|l|l|l|l|}
\hline \multicolumn{1}{|c|}{} & $\mathrm{n}$ & $\%$ \\
\hline Docente & Instituto Politécnico de Bragança & 45 & $23.8 \%$ \\
\cline { 2 - 4 } & Instituto Politécnico de Leiria & 59 & $31.2 \%$ \\
\cline { 2 - 4 } & Instituto Politécnico de Lisboa & 1 & $0.5 \%$ \\
\cline { 2 - 4 } & $\begin{array}{l}\text { Instituto Politécnico de Por- } \\
\text { talegre }\end{array}$ & 35 & $18.5 \%$ \\
\cline { 2 - 4 } & Instituto Politécnico de Santarém & 27 & $14.3 \%$ \\
\cline { 2 - 4 } & $\begin{array}{l}\text { Instituto Politécnico de Viana do } \\
\text { Castelo }\end{array}$ & 5 & $2.6 \%$ \\
\cline { 2 - 4 } & Instituto Politécnico de Viseu & 12 & $6.3 \%$ \\
\cline { 2 - 4 } & Universidade de Aveiro & 1 & $0.5 \%$ \\
\cline { 2 - 4 } & Universidade dos Açores & 4 & $2.1 \%$ \\
\cline { 2 - 4 } & Feminino & 126 & $66.7 \%$ \\
\cline { 2 - 4 } & Masculino & 63 & $33.3 \%$ \\
\hline Pessoal não & Instituto Politécnico de Bragança & 12 & $9.9 \%$ \\
\cline { 2 - 4 } & Instituto Politécnico de Leiria & 49 & $40.5 \%$ \\
\cline { 2 - 4 } & $\begin{array}{l}\text { Instituto Politécnico de Por- } \\
\text { talegre }\end{array}$ & 24 & $19.8 \%$ \\
\cline { 2 - 4 } & Instituto Politécnico de Santarém & 24 & $19.8 \%$ \\
\cline { 2 - 4 } & $\begin{array}{l}\text { Instituto Politécnico de Viana do } \\
\text { Castelo }\end{array}$ & 3 & $2.5 \%$ \\
\cline { 2 - 4 } & Instituto Politécnico de Viseu & 7 & $5.8 \%$ \\
\cline { 2 - 4 } & Universidade dos Açores & 2 & $1.7 \%$ \\
\cline { 2 - 4 } & Feminino & 91 & $75.2 \%$ \\
\cline { 2 - 4 } & Masculino & 30 & $24.8 \%$ \\
\hline Estudante & Instituto Politécnico de Bragança & 133 & $31.5 \%$ \\
\cline { 2 - 4 } & Instituto Politécnico de Leiria & 166 & $39.3 \%$ \\
\cline { 2 - 4 } & $\begin{array}{l}\text { Instituto Politécnico de Por- } \\
\text { talegre }\end{array}$ & 8 & $1.9 \%$ \\
\cline { 2 - 4 } & Instituto Politécnico de Santarém & 66 & $15.6 \%$ \\
\cline { 2 - 4 } & $\begin{array}{l}\text { Instituto Politécnico de Viana do } \\
\text { Castelo }\end{array}$ & 22 & $5.2 \%$ \\
\cline { 2 - 4 } & Instituto Politécnico de Viseu & 10 & $2.4 \%$ \\
\cline { 2 - 4 } & Instituto Politécnico do Porto & 1 & $0.2 \%$ \\
\cline { 2 - 4 } & Universidade dos Açores & 16 & $3.8 \%$ \\
\cline { 2 - 4 } & Feminino & $23.9 \%$ \\
\hline
\end{tabular}

Como instrumento de recolha de dados utilizou-se um questionário online, composto por uma parte com questões socioprofissionais, uma segunda parte com o Oxford Happiness Questionnaire (Hills \& Argyle, 2002), um questionário de autorrelato de 29 itens, respondidos numa escala de Likert de 6 pontos, e a terceira parte com a Escala de Ansiedade, Depressão e 
Stress (Lovibond \& Lovibond, 1995) na sua versão portuguesa (Ribeiro, Honrado e Leal, 2004), uma escala de autorrelato de 21 itens, respondidos numa escala de Likert de 4 pontos, medindo as dimensões Ansiedade, Depressão e Stress, cada uma com 7 itens. O tratamento de dados foi efetuado através do software estatístico IBM SPSS, versão 23 para macOS.

\section{RESULTADOS}

Para dar resposta ao primeiro objetivo do presente estudo "identificar os níveis de felicidade, ansiedade, depressão e stress dos docentes, pessoal não docente e estudantes do ensino politécnico", procedeu-se a uma análise descritiva dos dados, sendo apresentados na Tabela 2, as pontuações médias e respetivos desvios padrão para as quatro dimensões sob estudo: stress, ansiedade, depressão e felicidade, apresentadas pelos três grupos da nossa investigação: docentes, pessoal não docente e estudantes.

Tabela 2 - Médias e DP por dimensão por situação ocupacional

\begin{tabular}{|l|l|l|l|l|l|}
\hline & & Stress & $\begin{array}{l}\text { Ansie- } \\
\text { dade }\end{array}$ & $\begin{array}{l}\text { De- } \\
\text { pressão }\end{array}$ & $\begin{array}{l}\text { Felici- } \\
\text { dade }\end{array}$ \\
\hline \multirow{4}{*}{ oocente } & $\mathrm{M}$ & 5.94 & 2.96 & 3.21 & 4.26 \\
\cline { 2 - 6 } & $\mathrm{DP}$ & 4.109 & 3.534 & 3.667 & .662 \\
\cline { 2 - 6 } & $\mathrm{n}$ & 189 & 189 & 189 & 189 \\
\hline \multirow{3}{*}{$\begin{array}{l}\text { Pessoal não } \\
\text { docente }\end{array}$} & $\mathrm{M}$ & 6.74 & 4.13 & 4.62 & 4.01 \\
\cline { 2 - 6 } & $\mathrm{DP}$ & 4.208 & 3.926 & 4.648 & .670 \\
\cline { 2 - 6 } & $\mathrm{n}$ & 121 & 121 & 121 & 121 \\
\hline \multirow{4}{*}{ Estudante } & $\mathrm{M}$ & 7.57 & 4.98 & 5.14 & 3.93 \\
\cline { 2 - 6 } & $\mathrm{DP}$ & 4.881 & 4.462 & 4.963 & .742 \\
\cline { 2 - 6 } & $\mathrm{n}$ & 422 & 422 & 422 & 422 \\
\hline \multirow{3}{*}{ Total } & $\mathrm{M}$ & 7.02 & 4.32 & 4.56 & 4.03 \\
\cline { 2 - 6 } & $\mathrm{DP}$ & 4.632 & 4.236 & 4.675 & .724 \\
\cline { 2 - 6 } & $\mathrm{n}$ & 732 & 732 & 732 & 732 \\
\hline
\end{tabular}

Conforme pode ser observado pelos dados apresentados na Tabela 2, são os docentes que apresentam níveis mais baixos, em média, para as dimensões stress, ansiedade e depressão e o valor mais alto no que concerne a felicidade. $\mathrm{O}$ grupo que apresenta piores resultados em todas as dimensões são os estudantes, sendo que para as dimensões stress, ansiedade e depressão os resultados neste grupo são considerados "ligeiros", numa classificação que tem cinco níveis: normal; ligeiro; moderado; severo; e extremamente severo (Psychology Foundation of Australia, 2018).
Também para o pessoal não docente, os níveis de ansiedade e depressão caem na classificação de "ligeiro". Para medir a confiabilidade dos dados recolhidos, em relação às quatro dimensões sob estudo, foram calculados os alfas de Cronbach, sendo que os resultados apresentados na Tabela 3 mostram que as quatro dimensões mostram confiabilidade boa a excelente (>.90) (Cronbach, 1988).

Tabela 3 - Confiabilidade das dimensões

\begin{tabular}{|l|l|}
\hline & $\alpha$ \\
\hline Ansiedade & .863 \\
\hline Depressão & .910 \\
\hline Stress & .893 \\
\hline Felicidade & .916 \\
\hline
\end{tabular}

Para dar resposta ao segundo objetivo: identificar possíveis diferenças entre grupos, procedeu-se a uma análise inferencial.

Optou-se por aplicar o teste não-paramétrico de Kruskal-Wallis e Mann-Whitney, uma vez que, conforme demonstrado pelos testes de normalidade na Tabela 4, os dados da amostra apresentam uma distribuição não normal $($ sig<.05).

Tabela 4 - Testes de normalidade

\begin{tabular}{|c|c|c|c|c|c|c|}
\hline & \multicolumn{3}{|c|}{ Kolmogorov-Smirnova } & \multicolumn{3}{|c|}{ Shapiro-Wilk } \\
\hline & Estatística & $\mathrm{gl}$ & Sig. & Estatística & $\mathrm{gl}$ & Sig. \\
\hline Stress & .106 & 732 & .000 & .958 & 732 & .000 \\
\hline Ansiedade & .154 & 732 & .000 & .873 & 732 & .000 \\
\hline Depressao & .177 & 732 & .000 & .855 & 732 & .000 \\
\hline Felicidade & .036 & 732 & .026 & .992 & 732 & .000 \\
\hline
\end{tabular}

a. Correlação de Significância de Lilliefors

Optou-se por testes não-paramétricos seguindo as mais recentes recomendações a nível de tratamentos estatístico (Bishara \& Hittner, 2012; Ghasemi \& Zahediasl, 2012).

Através dos testes de Kruskal-Wallis, foi possível identificar que, para todas as dimensões, existiam diferenças estatisticamente significativas entre pelo menos um par de grupos. Para identificar os pares onde essas diferenças eram um facto, aplicaram-se testes de Mann-Whitney, sendo os resultados apresentados na Tabela 5. 
Tabela 5 - Testes de Mann-Whitney por

Dimensão e Situação

\begin{tabular}{|l|l|l|l|}
\hline Dimensão & Situação & $\mathrm{M}$ & $\mathrm{p}$. \\
\hline \multirow{4}{*}{ Stress } & Docente & 5.94 & \multirow{3}{*}{.000} \\
\cline { 2 - 3 } & Estudante & 7.57 & \\
\hline \multirow{4}{*}{ Ansiedade } & Docente & 2.96 & \multirow{3}{*}{.004} \\
\cline { 2 - 3 } & Estudante & 4.98 & \\
\cline { 2 - 3 } & Docente & 2.96 & \multirow{2}{*}{.000} \\
\cline { 2 - 3 } & Pessoal não docente & 4.13 & \\
\hline \multirow{5}{*}{ Felicidade } & Docente & 3.21 & \multirow{2}{*}{.009} \\
\cline { 2 - 3 } & Estudante & 5.14 & \\
\cline { 2 - 3 } & Docente & 3.21 & \multirow{2}{*}{.000} \\
\cline { 2 - 3 } & Pessoal não docente & 4.62 & \\
\hline & Docente & 4.26 & \multirow{2}{*}{.000} \\
\cline { 2 - 3 } & Estudante & 3.93 & \\
\cline { 2 - 3 } & Docente & 4.26 & \multirow{2}{*}{.003} \\
\cline { 2 - 3 } & Pessoal não docente & 4.01 & \\
\hline
\end{tabular}

Conforme observável na Tabela 5, as diferenças identificadas no nível de stress, são estatisticamente significativas para a combinação docente-estudantes $(\mathrm{p}<.001)$. No que concerne à dimensão ansiedade, as diferenças em valores são estatisticamente significativas para os pares docente-estudante $(\mathrm{p}=.004)$ e docente-pessoal não docente $(\mathrm{p}<.001)$. Em relação à dimensão depressão, os pares docente-estudante $(\mathrm{p}=.009)$ e docente-pessoal não docente $(\mathrm{p}<.001)$, são os que apresentam diferenças estatisticamente significativas. Finalmente, na dimensão felicidade são os pares docente-estudante $(\mathrm{p}<.001)$ e docente-pessoal não docente $(p=003)$, que apresentam diferenças estatisticamente significativas.

\section{DISCUSSÃO}

Tal como noutros estudos, verificou-se uma relação entre os níveis de ansiedade, depressão e stress e o nível de felicidade dos indivíduos (Cipresso, Serino, \& Giuseppe, 2014; Lyubomirsky et al., 2006; Schütz et al., 2013; Tarrahi \& Nasirian, 2017) Também no que diz respeito aos estudantes, os nossos resultados estão em concordância com os de vários estudos existentes, onde igualmente foram apresentados níveis acima do recomendado para as dimensões ansiedade, depressão e stress (Galvão, Noné e Gomes, 2016; Galvão, Pinheiro, Gomes e Ala, 2017). Vários estudos internacionais encontraram resultados similares com níveis baixos de felicidade entre os estudantes do ensino superior, como é o caso no presente estudo (Stewart, Watson, Clark, Ebmeier, \& Deary, 2010; Tarrahi \& Nasirian, 2017).

\section{CONCLUSÕES}

Verifica-se que em geral os docentes têm níveis de ansiedade, depressão e stress baixos e um nível de felicidade alto, ao contrário dos estudantes, que apresentam níveis consideravelmente piores nas quatro dimensões sob estudo, tornando-se evidente a importância que deve ser dada ao reforço da capacidade de resposta dos estabelecimentos de ensino superior na vertente das consultas de psicologia. Propomos que este estudo seja aprofundado para identificar as possíveis causas que provocam o baixo nível de felicidade e os altos níveis de ansiedade, depressão e stress dos estudantes, por forma a poder recomendar medidas a implementar.

\section{IMPLICAÇÕES PARA A PRÁTICA CLÍNICA}

Espera-se que este estudo possa contribuir para o conhecimento das necessidades desta população, em matéria de felicidade, depressão, ansiedade e stress, para, de alguma forma se poder refletir acerca de possíveis intervenções futuras. As instituições de ensino (neste caso superior) deverão encetar o delineamento de estratégias para melhorar, ou se possível reverter, os dados obtidos neste estudo.

Só fortalecendo o conhecimento baseado na evidência científica é possível desenvolver ações de promoção da saúde em geral e prevenção da doença mental em particular.

Os resultados encontrados sugerem a necessidade de apoio psicológico como promotor do bem-estar e da saúde mental positiva, pelo que a existência de um gabinete clinico nas respetivas instituições de ensino é uma aposta válida.

\section{REFERÊNCIAS BIBLIOGRÁFICAS}

Bishara, A. J., \& Hittner, J. B. (2012). Testing the significance of a correlation with nonnormal data: Comparison of Pearson, Spearman, transformation, and resampling approaches. Psychological Methods, 17(3), 399-417. Doi: 10.1037/a0028087 
Cipresso, P., Serino, S., \& Giuseppe, R. (2014). The pursuit of happiness measurement: A psychometric model based on psychophysiological correlates. Scientific World Journal, 2014. Doi: 10.1155/2014/139128

Cronbach, L. J. (1988). Internal consistency of tests: Analyses old and new. Psychometrika, 53(1), 63-70. Doi: $10.1007 / B F 02294194$

Galvão, A., Noné, A. R., \& Gomes, M. J. (2016). Alcoholism and coping strategies among IPB students. In S. Jesus, Proceedings of the International Congress on Interdisciplinarity in Social and Human Sciences (165-171). Algarve: University of Algarve - CIEO Research Centre for Spatial and Organizational Dynamics

Galvão, A., Pinheiro, M., Gomes, M. J., \& Ala, S. (2017). Ansiedade, Stress e Depressão Relacionados Com Perturbações do Sono-Vigília e Consumo de Álcool em Alunos do Ensino Superior. Revista Portuguesa de Enfermagem de Saúde Mental, 5(spe5), 8-12. Doi: 10.19131/rpesm.0160

Ghasemi, A., \& Zahediasl, S. (2012). Normality tests for statistical analysis: A guide for non-statisticians. International Journal of Endocrinology and Metabolism, 10(2), 486-489.Doi: 10.5812/ijem.3505

Hills, P., \& Argyle, M. (2002). The Oxford Happiness Questionnaire: A compact scale for the measurement of psychological well-being. Personality and Individual Differences, 33(7), 1073-1082. Doi: 10.1016/S01918869(01)00213-6

Jalloh, A., Flack, T., \& Chen, K. (2014). Measuring Happiness: Examining Definitions and Instruments. Illuminare, 12(1), 59-67. Disponível em: https:// scholarworks.iu.edu/journals/index.php/illuminare/ article/view/3635

Keramati, M. R. (2014). A comparison of health-related quality of life and job satisfaction in physically active and sedentary faculty members. International Journal of Education and Applied Sciences, 1(2), 6877.
Lovibond, P., \& Lovibond, S. (1995). The structure of negative emotional states: Comparison of the Depression Anxiety Stress Scales (DASS) with the Beck Depression and Anxiety Inventories. Behaviour Research and Therapy, 33(3), 335-343. Doi: 10.1016/00057967(94)00075-u

Lu, L., \& Argyle, M. (1994). Leisure satisfaction and happiness as a function of leisure activity. The Kaohsiung journal of medical sciences, 10(2), 89-96. Doi:10.1016/0005-7967(94)00075-u

Lyubomirsky, S., Tkach, C., \& DiMatteo, M. R. (2006). What are the differences between happiness and selfesteem? Social Indicators Research, 78(3), 363-404. Doi: 10.1007/s11205-005-0213-y

Psychology Foundation of Australia. (2018). Depression Anxiety Stress Scales - DASS. Disponível em: http://www2.psy.unsw.edu.au/dass/

Ribeiro, J. L. P., Honrado, A., \& Leal, I. (2004). Contribuição para o estudo da adaptação portuguesa das Escalas de Ansiedade, Depressão e Dtress (EADS) de 21 itens de Lovibond e Lovibond. Psicologia, Saúde \& Doenças, 5(2), 229-239.Disponível em: http://www.scielo.mec.pt/scielo.php?script=sci_ abstract\&pid $=$ S1645-00862004000200007\&lng=pt\&n $\mathrm{rm}=\mathrm{iso}$

Schütz, E., Sailer, U., Al Nima, A., Rosenberg, P., Andersson Arntén, A.-C., Archer, T., \& Garcia, D. (2013). The affective profiles in the USA: happiness, depression, life satisfaction, and happiness-increasing strategies. PeerJ, 1, e156. Doi: 10.7717/peerj.156

Stewart, M. E., Watson, R., Clark, A., Ebmeier, K. P., \& Deary, I. J. (2010). A hierarchy of happiness? Mokken scaling analysis of the Oxford Happiness Inventory. Personality and Individual Differences, 48(7), 845-848. Doi: 10.1016/j.paid.2010.02.011

Tarrahi, M. J., \& Nasirian, M. (2017). Happiness and risk behaviours in freshman students of Khorramabad Universities. Iranian Journal of Psychiatry and Behavioral Sciences, 11(2). Doi: 10.5812/ijpbs. 7345 


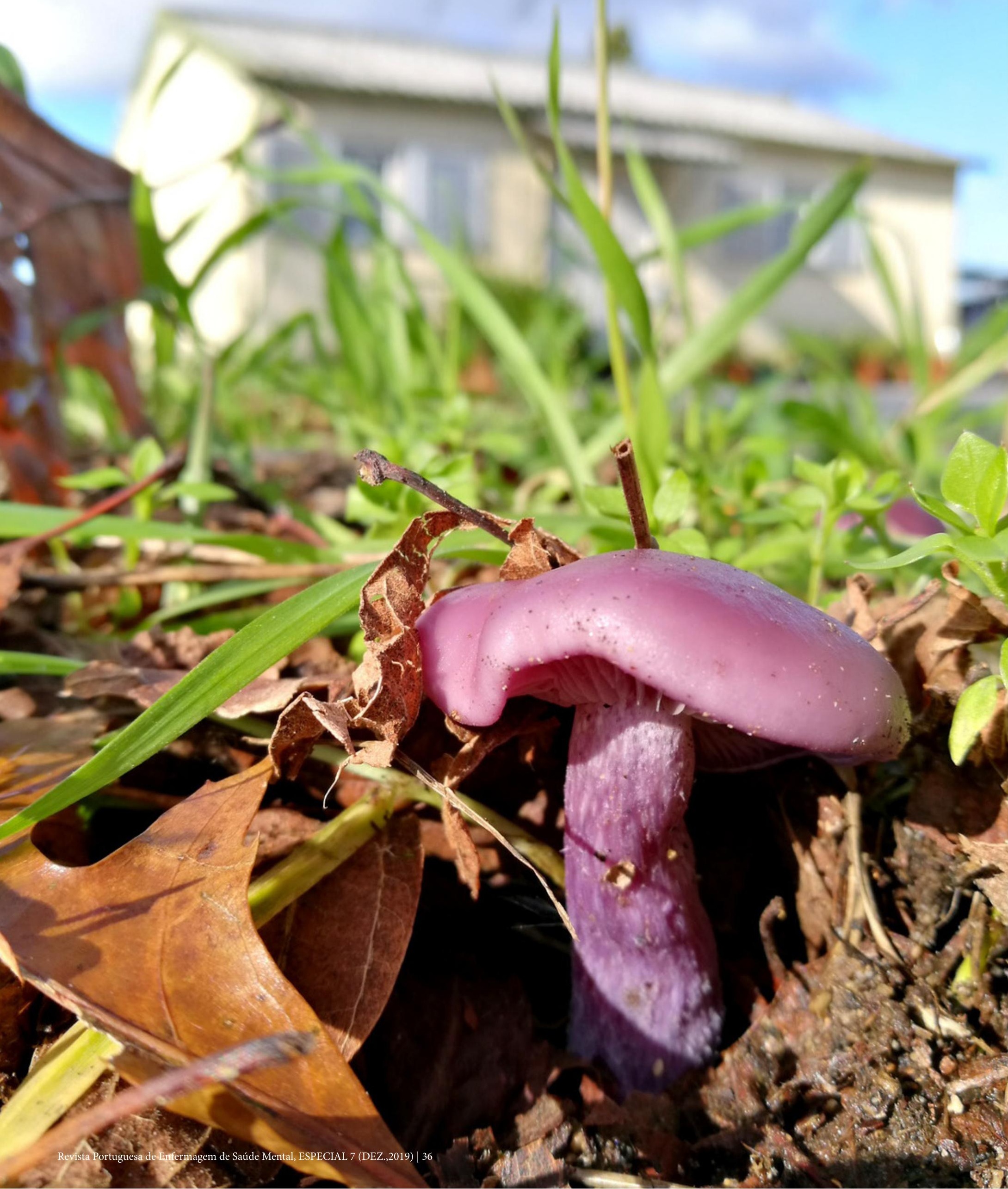

